\title{
Mechanical Behaviors Study of Epoxy Resins Reinforced with Nano-SiO 2 Particles
}

\author{
Jian Zheng, Xiong Chen, Deng Jia, Xin Tong \\ School of Mechanical Engineering, Nanjing University of Science and Technology, \\ Nanjing, 210094, China \\ email: zhengjian@njust.edu.cn
}

Keywords: Mechanical Performance; Epoxy Resin; Nanoparticles; Elastic Modulus

\begin{abstract}
Epoxy resin composites filled with nanoparticles are widely used for electrical devices such as insulators, VLSI package and rocket engine shell materials. An experimental study on the mechanical property of $\mathrm{SiO}_{2}$-filled epoxy resin composite was carried out by the electronic universal testing machine. The prepared experimental specimens were made of three nano- $\mathrm{SiO}_{2}$ particles(15nm, 30nm and 50nm) at different weight fractions (2\%, 4\% and $8 \%)$. Mechanical characteristics including yield strength limit, elastic modulus were obtained. The experimental results showed the addition of nanoparticles will improve the mechanical performance of the epoxy resin composite. Its improvement was better for yield limit with the nanoparticles content of $2 \%$, and for yield limit and elastic modulus with the nanoparticles radius of 30nm.
\end{abstract}

\section{Introduction}

Epoxy resin(abbreviation:EP) composites filled with nanoparticles are a widely used material system for electrical devices such as insulators, electrorheological fluids, VLSI package and rocket engine shell materials. There is a keen interest to clarify the relevance of the primary properties of $\mathrm{SiO}_{2}$ particles on the rheological characteristics of the composite system[1-4]. The study found that, the mechanical property of epoxy resin can be improved by the addition of nanoparticles in the epoxy resin. Thus making further improvement and research on the mechanical property of this material is the most important for the design and manufacturing of solid rocket engine.

The contribution of particle structure to the viscosity of a $\mathrm{SiO}_{2}$-filled epoxy resin system was revealed as one of the intrinsic properties of $\mathrm{SiO}_{2}$ by using transparent optical microscopy. This was found to be a reliable tool for identifying particle-aggregated structures and their peculiar viscosity characteristics. However, their rheological properties remained unclear solely from the well-mentioned filler primary properties based on the characterization of the $\mathrm{SiO}_{2}$-filled epoxy resin system: median diameters, size distributions, specific surface areas and surface hydroxyl group structures[5].

Nano-sized copoly particles were added at different contents to improve the toughness of diglycidyl ether of bisphenol A epoxy resin (ER) using piperidine as a curing agent. To compare the adhesion strength of different adherents utilizing both modified and unmodified epoxy adhesive, the lap shear strength (LSS) test was measured as a function of elastomeric nanoparticles (ENP) amount. Scanning electron microscopy (SEM) and FTIR were used to investigate the interface morphology and chemical composition of adherent and epoxy adhesive. The result indicated that the adhesion strength was increased dramatically by addition of nanoparticles compared with that of pure epoxy adhesive. The highest adhesion strength was obtained with $20 \mathrm{wt} \%$ elastomeric nanoparticles. It was found that reinforcement with nanoparticles improved the fracture toughness[6].

Epoxy formulations containing mixtures of two different size distributions of silica-based particles (micron-size and nanometer-size) were explored for possible synergistic toughening effects. The influence of bimodal particle size distribution and silica particle content on the glass transition temperature (Tg), coefficient of thermal expansion (CTE), Young's modulus (E), tensile yield stress (sy), and fracture toughness were investigated. Interestingly, fracture toughness improved by approximately $30 \%$ when mixtures of microparticles and nanoparticles were used. The 
improvements in toughness were higher when the volume fraction of microparticles was less than the volume fraction of nanoparticles (overall filler content was fixed at $10 \mathrm{vol} \%$ in this study)[7].

Mechanical properties of toughened epoxy resin with two nano particles sizes of $\mathrm{TiO}_{2}(17 \mathrm{~nm}$ and $50 \mathrm{~nm}$ ) at different weight fractions (1\%, 3\%, 5\% and 10\%) were investigated and compared to that of submicron particles at $220 \mathrm{~nm}$. The composites were characterized by tensile, flexural, pull-off and abrasion tests, followed by X-ray photoelectron spectroscopy and scanning electron microscopy of the fracture surfaces. The investigated epoxy depicted high performance epoxy systems formulated with low molecular weight epoxy resin and diethyltoluenediamine as a curing agent[8].

The thermo-mechanical behavior of epoxy resins/nano- $\mathrm{Al}_{2} \mathrm{O}_{3}$ composites including the curing behavior, thermal stability, dynamic mechanical properties and thermal mechanical properties. The DSC curve peak temperature of the composites was decreased by the addition of nano- $\mathrm{Al}_{2} \mathrm{O}_{3}$. The thermal stability of the composites was similar to that of the neat epoxy resins. Dynamic mechanical analysis (DMA) indicated the glass transition temperature of the composites to be approximately $11 \mathrm{C}$ higher than that of the neat epoxy resins. The coefficient of thermal expansion (CTE) of the composites decreased with increasing nano- $\mathrm{Al}_{2} \mathrm{O}_{3}$ content[9].

An experimental study on the mechanical property of $\mathrm{SiO}_{2}$-filled epoxy resin composite was carried out in this paper. The prepared experimental specimens were made of three nanoparticles sizes of $\mathrm{SiO}_{2}(15 \mathrm{~nm}, 30 \mathrm{~nm}$ and $50 \mathrm{~nm})$ at different weight fractions (2\%, 4\% and $\left.8 \%\right)$. The constant velocity tensile test for the specimens was undertaken by the electronic universal testing machine, and its mechanical characteristics including yield strength limit, elastic modulus were obtained. The experimental results can provide the technical support for the design and manufacturing of solid rocket engine.

\section{Experiment Preparation}

\subsection{Sample preparation procedure}

Commercialized spherical SiO2 particles(Chengdu West Reagent Co., Ltd.) used practically in the package material field were provided as filler particles. Epoxy resin (Nantong Xingchen synthetic material Co., Ltd.) was utilized as solvent for the composite system. The $\mathrm{SiO} 2$ particles $(15 \mathrm{~nm}, 30 \mathrm{~nm}, 50 \mathrm{~nm})$ were filled into the epoxy resin with a solid loading of 2,4,8 mass\%. The composite system was mixed with curing agent (low molecular 650-eurelon; Nantong Xingchen synthetic material Co., Ltd.) for 5 min at 600 r.p.m. rotation speeds.

The manufacture procedure of the specimen are as follows: (1)Put quantify epoxy resin in a disposable cup. (2) Heat the epoxy resin to 80C, then add nanoparticles and stir until there is no nanoparticles agglomeration. (3) Add eurelon curing agent, assure that eurelon and epoxy resin volume ratio is $1: 4$, then keep string for 5 minute. (4) Put the prepared reagent in the incubator, and keep warm for 10 minutes at 80C, then exhaust air and pour it in the prefabricated mould. (5)Place the specimens in the lab for curing. The cure time of the specimens is 35 days. (6) Finish the specimens on the multi-purpose machine to meet the size requirement in figure 1.

2.2 Experimental equipment and relevant standard

The experiment was performed by the electronic universal testing machineis with $1 \mathrm{~mm} / \mathrm{min}$ tensile rate at the room temperature of 26Cand the humidity of 32\% on the basis of GB/T228-2002 standard. All the slablike specimens were manufactured in the shape of "dog-bone", that is, the ends of the specimens are sross and the rest is relatively slimsy, the transitional region is the shape of $\operatorname{arc}[10]$. The geometry of the specimen is shown in figure 1 .

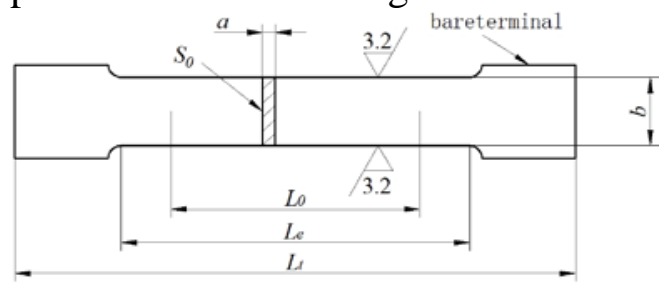

Fig.1. The geometry of the specimen 


\section{Mechanical Performance Experiment}

\subsection{Experimental data reduction}

The investigation object of the experiment is the epoxy resin reinforced with $\mathrm{SiO}_{2}$ nanoparticles. It's a kind of hard brittle material in essence, and therefor the material's ultimate strength is an important mechanical property obtained from the experiment. The ultimate strength curve of the material by the tensile test are as shown in the figure 2 and figure 3 .

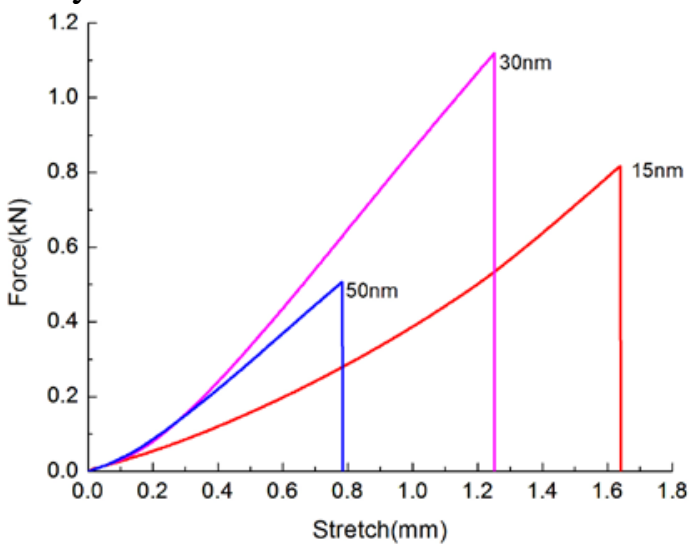

Fig.2. The tensile curves of the specimens with different particle size

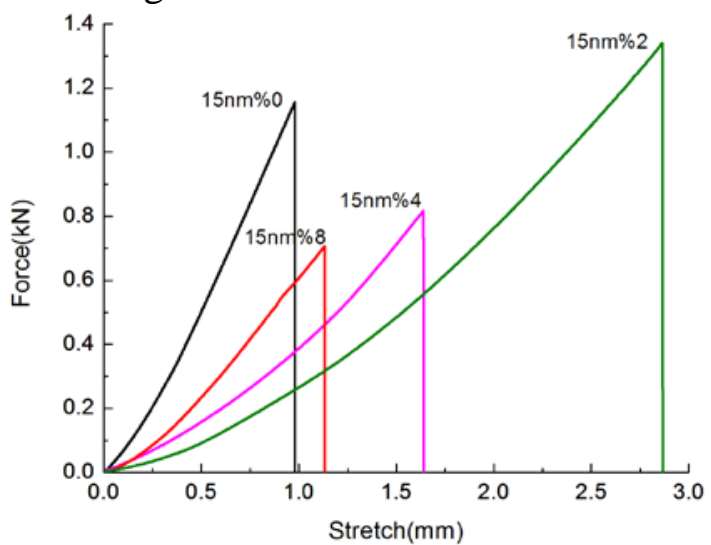

Fig.3. The tensile curves of the specimens with different particle content

As we can see from figure 3, for different nanoparticles, the shape of the curve showed different change trends. Simply speaking, the larger content of nanoparticles, the material's stiffness decrease, and the linearity property become worse. Moreover, with the increase of the content of nanoparticles, the material will present hyperelastic deformation obviously. In addition, the radius of the nanoparticles will also affect the property of epoxy resin significantly.

The increasing of content of the nanoparticles will cause the presentation of hyperelastic of the material. The possible reasons as follows: after the intensive mixing of the nanoparticles and specimens, the nanoparticles will firmly bonded to the substrates. If the deformation of the specimen is small, the force between the particles and substrates is small, but while the deformation is big, the particles will pull the substrates that present separation trend, limit the deformation of substrates. Thus, when the stress in the specimen is small, due to the presence of nanoparticles, the content of substrates is lower, in a cross section, most of the region is filled with particles. Someone believe that the content of the nanoparticles is only $2 \%, 4 \%$ and $8 \%$, as a consequence, its influence on the result is very low, thus, it will not cause the phenomenon that the stiffness of the specimens in the initial stage lower than the specimens that without nanoparticles. But from the experiment,we observed that, although the mass fraction of the nanoparticles is very low, its density is minimum, so it's volume content is very high . Thus, less weight of nanoparticles will cause obvious change in the specimens, and explained the low stiffness in the initial stage.

With the displacement increase, the deformation of the specimen become larger. Nanoparticles will give full play to its adhesive ability with the substrate, and produce a large force to prevent deformation and displacement. The nanoparticles is rigid particles, as a consequence, the stiffness of the specimen increased, and present a trend to increase continuously, thus, showed a hyperelastic performance in mechanical property. But with the increase of displacement, the reversion function of the stiffness particles reaches the ultimate, then the material present a obvious linear property until the specimen was broken up.

3.2 The determination of yield limit and elastic modulus

The maximum load bearing force could be obtained from force-displacement curve and then,yield stress of this material, can be calculated according to formula (1):

$$
\sigma_{b}=\frac{F_{b}}{A}
$$

The value of namely the cross-sectional area,could be gained utilizing following method: measuring the cross-sectional area of three different locations in the middle sample and then 
choosing their minimum value as final value in formula (1).

$$
\begin{array}{rl}
A_{i}=b_{i} & * h_{i}(i=1,2,3) \\
& A=\min \left(A_{1}, A_{2}, A_{3}\right)
\end{array}
$$

Elastic modulus is one of the important performance parameters of material. The value of elastic modulus reflects material's stiffness and capacity of resisting deformation. In this paper, 'inverse point translation method' and 'slope method' has been applied to get this material's elastic modulus. 'Imaginary elastic modulus', associating with slope, can be expressed as[11]:

already known:

$$
k=\frac{\Delta F}{\Delta l}
$$

and:

$$
E=\frac{\Delta \sigma}{\Delta \varepsilon}=\frac{\Delta F / A}{\Delta l / l_{0}}=\frac{\Delta F}{\Delta l} \frac{l_{0}}{A}=k \frac{l_{0}}{A}
$$

The key of acquiring elastic modulus is the cross-sectional area $A$, and gauge length $l_{0}$. $A$ value derives from formula (5) after getting the value of $A_{1}, A_{2}, A_{3}$ based on formula (2).

$$
A=\frac{1}{3}\left(A_{1}+A_{2}+A_{3}\right)
$$

Gauge length $l_{0}$, is determined by formula (6) .

$$
l_{0}=\frac{1}{2}\left(l_{t}+l_{c}\right)
$$

3.3 The effect of nanoparticles content and radius on elastic modulus

The effects of the nano- $\mathrm{SiO}_{2}$ fraction content on the elastic modulus of the epoxy resin composite investigated are shown in Figure 4. The strength limit of epoxy resin composite reaches its maximum value when nanoparticles content is $2 \%$. With the increase of nanoparticles content, the ultimate strength of the material decrease. On the one hand, the addition of nanoparticles can enhance its ultimate strength. On the other hand, with the increase of nanoparticles content, the agglomeration of nanoparticles and a small amount of bubbles produced in the internal of epoxy resin composite, will affect its mechanical performance. With the increase of nanoparticles content, the elastic modulus of the epoxy resin composite decrease.

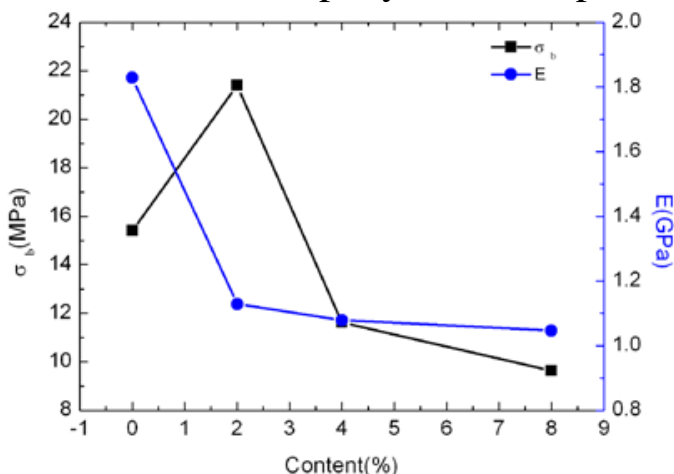

Fig.4. Stress limit and elastic modulus of the specimens with different particle content

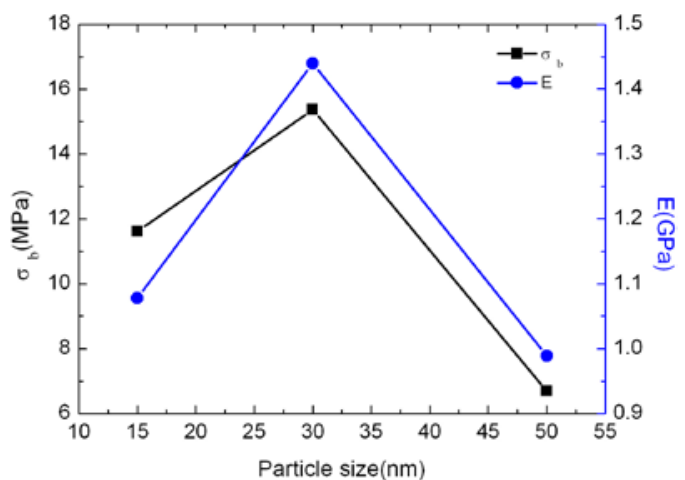

Fig.5. Stress limit and elastic modulus of the specimens with different particle size

The effects of the nano- $\mathrm{SiO}_{2}$ particles sizes on the elastic modulus of the epoxy resin composite investigated are shown in Figure 5. The strength limit of material reaches its maximum value when nanoparticles radius is 30nm, which could be clearly observed in figure 5. Nanoparticles' capacity of pulling matrix is limited as their radius are too small. As a result, the mechanical property is not very good, but closes to $30 \mathrm{~nm}$. However the strength limit of material will be immediately deteriorated due to large radius of nanoparticles.The existence of this phenomenon may attributed to reunion of nanoparticles because of their large radius. Moreover, the ability of nanoparticles to pull the matrix will also be affected.

According to calculation of elastic modulus, the material's elastic modulus is the largest as nanoparticles' radius are 30nm. At this moment, the epoxy resin composite with particle radius of 
30nm exhibits good mechanical property.

\section{Conclusion}

(1)The addition of nanoparticles will improve the performance of the epoxy resin composite. This improvement is better as the content of nanoparticles is $2 \%$ for yield limit.

(2)Effect of nanoparticle radius of the strength and stiffness of the sample is also present.The experimental results show that, when the radius of the nanoparticles is $30 \mathrm{~nm}$, properties of the materials is relatively good. This phenomenon is rarely mentioned in previous research.

\section{References}

[1] T. K. Gupta and J. H. Jean, Principles of the development of a silica dielectric for microelectronics packaging, J. Mater. Res. 1999 (11) 243-263.

[2] M. K. McMurray and S. Amagi, The effect of time and temperature on flexural creep and fatigue strength of a silica particle filled epoxy resin, J. Mater. Sci. 1999 (34) 5927-5936.

[3] P. E. Wayne-Lougher, Ceramic application in chemical mechanical planarization, Am. Ceram. Soc. Bull. 1999 (78) 97-100 .

[4] H. Homma, Application of polymeric insulationmaterials for electric power facilities, Bull. Inst. Elect. Engng, Japan, 2000 (120) 152-155.

[5] Yasumasa Takao, Makio Naito and Hidehiro Kamiya, Contribution of particle structure to the viscosity of $\mathrm{SiO}_{2}$ Particle-filled epoxy resin composite systems, Advanced Powder Technol., 2001 12(1) 17-31.

[6] Sepideh Khoee, Narges Hassani, Adhesion strength improvement of epoxy resin reinforced with nanoelastomeric copolymer, Materials Science and Engineering A, 2010 (527) 6562-6567.

[7] Peerapan Dittanet, Raymond A. Pearson, Effect of bimodal particle size distributions on the toughening mechanisms in silica nanoparticle filled epoxy resin, Polymer, 2013 (54) 1832-1845.

[8] Hamad A. Al-Turaif, Effect of nano $\mathrm{TiO}_{2}$ particle size on mechanical properties of cured epoxy resin, Progress in Organic Coatings, 2010 (69) 241-246.

[9] Wei Jiang, Fan-Long Jin, Soo-Jin Park, Thermo-mechanical behaviors of epoxy resins reinforced with nano- $\mathrm{Al}_{2} \mathrm{O}_{3}$ particles, Journal of Industrial and Engineering Chemistry, 2012 (18) 594-596.

[10] GB/T 228-2002, The tensile test of metal materials at room temperature, 2002.

[11] Liu Hong-wen. Mechanics of Materials. Higher Education Press, 2004.6. 